\title{
Evaluation of diagnostic value of neutrophil to lymphocyte ratio in younger and older pediatrics suspect to acute appendicitis in Imam Hossein hospital
}

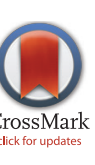

\author{
Azar Baradaran $^{\mathbb{D}}$, Azar Naimi ${ }^{\mathbb{D}}$, Elahe Pirpiran* ${ }^{(\mathbb{D}}$, Masoud Akhlaghi $^{\circledR}$ \\ Department of Pathology, School of Medicine, Isfahan University of Medical Sciences, Isfahan, Iran
}

Correspondence to:

Elahe Pirpiran,

Email: elahepirpiran@gmail.com

Received: 20 October 2020 Accepted: 7 December 2020 ePublished: 29 December 2020

Keywords: Diagnostic, Imam Hossein hospital, Neutrophil to lymphocyte, Appendicitis

\begin{abstract}
Introduction: Acute appendicitis in children is the most common acute surgical condition in children. Each year, 80000 children in the United States suffer from appendicitis.

Objectives: The aim of this study was to evaluate diagnostic value of neutrophil to lymphocyte ratio (NLR) in younger and older pediatrics suspect of acute appendicitis in Imam Hossein hospital.

Patients and Methods: This was a retrospective study conducted at Imam Hossein hospital from 2015-2017.

The study population was all children with suspected appendicitis who refer to Imam Hossein medical center in Isfahan. The sample size was 423 people. The collected data were imported into SPSS software version 22 and analyzed with appropriate statistical tests. The significance level in the present study was less than 0.05 .

Results: The average age of participants was $7.2 \pm 3.8$ years. In both study groups, it was observed that the mean NLR in the normal and reactive follicular groups was significantly lower than the appendicitis and complication groups $(P<0.05)$. The results of the present study showed that NLR diagnostic test can be a good predictor for the evaluation of appendicitis in both groups under 4 years and over 4 years. For more than 4 years' group; $\mathrm{AUC}=0.74, P<0.001$ and for less than 4 years' group; $\mathrm{AUC}=0.69, P<0.001$. For less than 4 years' group, the cut-off score for appendicitis diagnosis was 2.3. In this cut-off, the sensitivity and specificity were equal to $0.65 \%$ and $0.72 \%$. Additionally, for more than 4 years' group, the cut-off for the diagnosis of appendix was 3.5. In this cut-off, the sensitivity and specificity were equal to $0.73 \%$ and $67 \%$. Conclusion: This analysis demonstrates that NLR, in the context of appropriate clinical assessment of patients with a high a priori probability of appendicitis, has a greater diagnostic accuracy in supporting the diagnosis of appendicitis.
\end{abstract}

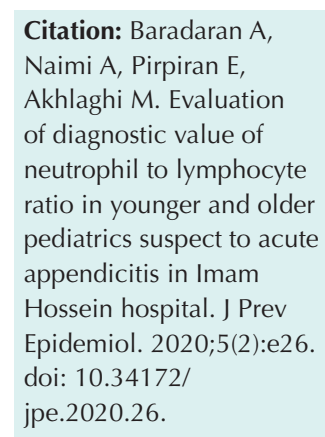

Citation: Baradaran A Naimi A, Pirpiran E, Akhlaghi M. Evaluation of diagnostic value of neutrophil to lymphocyte ratio in younger and older pediatrics suspect to acute appendicitis in Imam ossein hospital. J Prev doi: 10.34172 jpe. 2020.26

\section{Introduction}

Acute appendicitis in children is the most common acute surgical condition in children. Each year, 80000 children in the United States suffer from appendicitis. The incidence rate in children under 14 years of age is 4 per 1000 cases. The most common age of appendicitis in children is between 18-12 years old. It is rare (less than $5 \%$ ) at the age of less than 5 years and very rare (less than 1\%) at the age of less than 3 years. It is less common in boys than girls and more common in whites than blacks. The seasonal prevalence of appendicitis is often in spring and autumn. Mortality is rare in this disease. Despite radiological techniques in diagnosing appendicitis, it is still difficult to diagnose and there is a $20 \%$ risk of perforation (1).

Abdominal pain is one of the early symptoms of the disease that begins in the early hours after the onset of the disease. Nausea and vomiting occur in more than

\section{Key point}

In a study on 423 people with $7.2 \pm 3.8$ years old, we found that neutrophil to lymphocyte ratio (NLR) diagnostic test can be a good predictor for the evaluation of appendicitis.

$50 \%$ of patients and are often followed by abdominal pain within a few hours. Anorexia is a classic finding in acute appendicitis. Fever is mild in appendicitis (2).

Diagnosis of appendicitis is initially with clinical signs. Abdominal examination is mild in the early stages of appendicitis, and abdominal distension is seen in more advanced cases and perforation or obstruction of the small intestine. Local abdominal tenderness is the most reliable finding in the diagnosis of acute appendicitis (3).

On the other hand, children have little usable information due to lack of cooperation with the doctor and poor ability to speak, 
which leads to poor history. Examining their abdomen is very difficult and challenging, especially in patients younger than 4 years.

For complex appendicitis, C-reactive protein (CRP) has the highest diagnostic accuracy. Recently, some clinical and laboratory indices such as neutrophil/lymphocyte $(\mathrm{N} / \mathrm{L})$ ratio and pediatric appendicitis score (PAS) have been used in the timely and accurate diagnosis of appendicitis $(4,5)$. Appendicitis must first be based on clinical examination and clearly requires more systemic and specific markers of inflammation.

\section{Objectives}

Use of cut-off, white blood cell (WBC) and CRP makes it more sensitive to diagnose complicated appendicitis (6).

The use of these biomarkers alone or in combination with other biomarkers can help diagnose the disease, although the sensitivity and specificity of these tests have varied in different studies and have not been used independently. Due to the above information and the lack of an efficient article and the lack of consistent results in various studies on this subject, we considered it necessary to conduct a study in this field, to know more about the role of blood biomarker information in this disease. Therefore, the aim of this study was to evaluate diagnostic value of neutrophil to lymphocyte ratio (NLR) in younger and older pediatrics suspect of acute appendicitis in Imam Hossein hospital.

\section{Patients and Methods}

\section{Study design}

This was a retrospective study conducted at Imam Hossein hospital from 2015-2017. The study population was all children with suspected appendicitis who refer to Imam Hossein medical center in Isfahan.

\section{Inclusion and exclusion criteria}

Inclusion criteria: Children must be between one month and 18 years old and have electronic information in the hospital file.

Exclusion criteria: Lack of sufficient information in the patient's file.

\section{Sampling method and sample size}

The sampling method was a non-probabilistic sampling. Due to the sample size formula in diagnostic studies that were presented below, 95\% confidence interval and considering the diagnostic sensitivity of ultrasound in previous studies equal to $84.6 \%$ and the matching ratio was 0.43 and the error level was 0.05 , the sample size was 423 people.

$$
\text { Sample size }(n) \text { based on sensitivity }=\frac{Z_{1-\alpha / 2}^{2} \times S_{N} \times\left(1-S_{N}\right)}{L^{2} \times \operatorname{Pr} \text { evalence }}
$$

\section{Data collection}

In the beginning, patients who were suspected of having acute appendicitis and have been diagnosed by a specialist were imported to the study and the $\mathrm{CBC}$ of these patients, which includes the WBC count of these patients, was collected. Additionally, the amount of lymphocytes and neutrophils were imported in the data collection form. The pathology report of these patients was reviewed and the patients of each group are divided into three groups; acute, reactive, and complication appendicitis. Finally, the results of the above experiments will be compared between the three groups in each age group.

\section{Ethical issues}

The research followed the tenets of the Declaration of Helsinki. The institutional ethical committee at Isfahan University of Medical Sciences approved all study protocols (IR.MUI.MED.REC.1397.011). Accordingly, written informed consent was taken from all participants before any intervention.

\section{Statistical analysis}

The collected data were imported into SPSS software version 22 and analyzed with appropriate statistical tests. The significance level in the present study was less than 0.05 .

\section{Results}

A total of 423 participants with complete information in the present study were evaluated. The average age of participants was $7.2 \pm 3.8$ years. Among them, 107 participants were less than four years old and 316 people were more than 4 years old. Additionally, 248 participants were girls and 175 were boys. Independent $t$ test was used to compare the mean NLR in age and gender groups.

The results of comparing the means are given in Table 1. As the results of this table show, the mean NLR index in more than 4 years is significantly higher than under 4 years $(P<0.05)$. However, no significant difference was detected among the mean NLR between the gender (girl and boy) $(P>0.05)$.

In order to compare the mean NLR in normal, appendicitis, complication and reactive follicular groups in different age groups, analysis of variance was used. The results are given in Table 2 . As the results of Table 2 show, the mean NLR in the study groups and by age has a significant difference $(P<0.05)$.

In children under 4 years of age, after the Bonferroni post hoc test, it was observed that there was a significant

Table 1. Comparison of NLR means by age and gender in the present study

\begin{tabular}{llllll}
\hline NLR & & Number & Mean & SD & P value \\
\hline \multirow{2}{*}{ Age $(y)$} & $<4$ & 107 & 3.32 & 3.1 & \\
& $>4$ & 316 & 6.32 & 5.2 & $<0.001$ \\
\multirow{2}{*}{ Gender } & Girl & 248 & 5.79 & 5.4 & \\
& Boy & 175 & 5.23 & 4.2 & 0.2 \\
\hline
\end{tabular}

Due to the normality of the data, independent $t$ test was used. Significance level less than 0.05 are considered. 
Table 2. Comparison of mean NLR in normal, appendicitis, complication and reactive follicular groups in different age groups

\begin{tabular}{|c|c|c|c|c|c|c|}
\hline NLR & & Number & Mean & SD & $\mathbf{F}$ & $P$ value \\
\hline \multirow{4}{*}{$\begin{array}{l}\text { Less than } 4 \\
\text { years }\end{array}$} & Reactive follicular & 34 & 2.19 & 1.85 & \multirow{4}{*}{4.47} & \multirow{4}{*}{0.005} \\
\hline & Normal & 11 & 1.71 & 1.02 & & \\
\hline & Appendicitis & 55 & 4.20 & 3.75 & & \\
\hline & Complication & 7 & 4.48 & 3.26 & & \\
\hline \multirow{4}{*}{$\begin{array}{l}\text { More than } 4 \\
\text { years }\end{array}$} & Reactive follicular & 76 & 3.6 & 3.06 & \multirow{4}{*}{10.14} & \multirow{4}{*}{$<0.001$} \\
\hline & Normal & 1 & 1.1 & - & & \\
\hline & Appendicitis & 222 & 7.1 & 5.6 & & \\
\hline & Complication & 17 & 8.1 & 3.9 & & \\
\hline
\end{tabular}

Due to the homogeneity of variance and normality of the data, analysis of variance was used to analyze the data. Significance level less than 0.05 are considered.

difference in the mean NLR between the appendix with normal and reactive follicular groups, therefore this index in normal and reactive follicular groups They were significantly less than the appendicitis and complication group $(P<0.05)$.

In children older than 4 years, after the post hoc test, it was observed that the mean NLR in the normal and reactive follicular groups was significantly lower than the appendicitis and complication groups $(P<0.05)$.

Figure 1 shows the changes in the mean NLR trend in the appendicitis, normal, complication, and reactive follicular groups by age groups less than 4 years and more than 4 years. In addition, the mean NLR in the Complicated and appendicitis groups was significantly higher than the reactive follicular and normal groups.

The results related to diagnostic value of NLR and sensitivity and specificity in younger and older pediatrics suspect of acute appendicitis were given in Table 3. Besides, ROC curve diagrams for groups less than 4 years and more than a year were given separately in Figure 2A-2B.

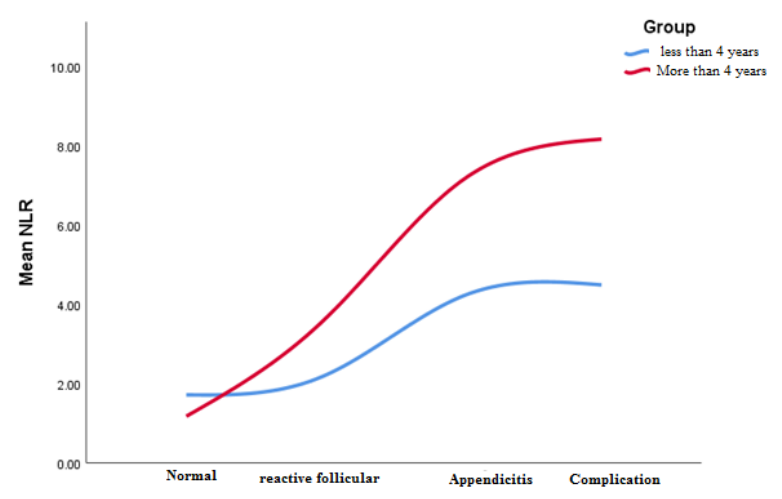

Figure 1. Changes in the mean NLR in the four groups of less than 4 years and more than 4 years
The results of the present study showed that NLR diagnostic test can be a good predictor for the evaluation of appendicitis in both groups fewer than 4 years and over 4 years. For more than 4 years' group; $\mathrm{AUC}=0.74, P<0.001$ and for less than 4 years' group; AUC $=0.69, P<0.001$.

As Table 3 shows, for less than 4 years' group, the cut-off score for appendicitis diagnosis was 2.3. In this cut-off, the sensitivity and specificity were equal to $0.65 \%$ and $0.72 \%$. Accordingly, for more than 4 years' group, the cut-off for the diagnosis of appendix was 3.5. In this cut-off, the sensitivity and specificity were equal to $0.73 \%$ and $67 \%$.

\section{Discussion}

Acute appendicitis is an inflammatory disease due to infection of appendix vermiformis. The causes of the disease are not well-detected exactly; however the etiology is assumed started by the obstruction of appendiceal lumen. Literature review show acute appendicitis is one of the most observed reason of surgical conditions, and also is the most common illness requiring abdominal surgery $(5,7)$.

Acute appendicitis is usually detected clinically. Indeed the most frequent inflammatory biomarker is white blood cells. Additionally CRP, interleukin-6, interleukin-10 may also be applied (1).

Many studies have been performed in order to diagnose acute appendicitis accurately, and rapidly. Goodman et al found NLR as a diagnostic test, for the diagnosis of acute appendicitis when greater than 3.5. They recognized high white blood cells in 298 individuals (79\%) with acute appendicitis, and also NLR 3.5 or greater than 3.5 was seen in 324 participants (88\%) with acute appendicitis, and the difference was found statistically considerable $(1,3)$. This finding is similar to the result of the present study. We showed NLR cut-off value as 3.5 for more than four years patients in applicable. Accordingly, we found,

Table 3. Diagnostic value of neutrophil to lymphocyte ratio in younger and older pediatrics suspect to acute appendicitis

\begin{tabular}{llllll}
\hline Diagnosis of NLR in appendicitis & AUC & Cut-off of NLR & Sensitivity & Specificity & LR+ \\
\hline Less than 4 years $(n=107)$ & 0.69 & $\leq 2.3$ & $0.65 \%$ & $0.72 \%$ & 0.48 \\
More than 4 years $(n=316)$ & 0.74 & $\leq 3.5$ & $73 \%$ & $67 \%$ & 0.40 \\
\hline
\end{tabular}

Note. $A \cup C=$ area under the receiver operative curve; $L R+=$ positive likelihood ratio; $L R-=$ negative likelihood ratios. 

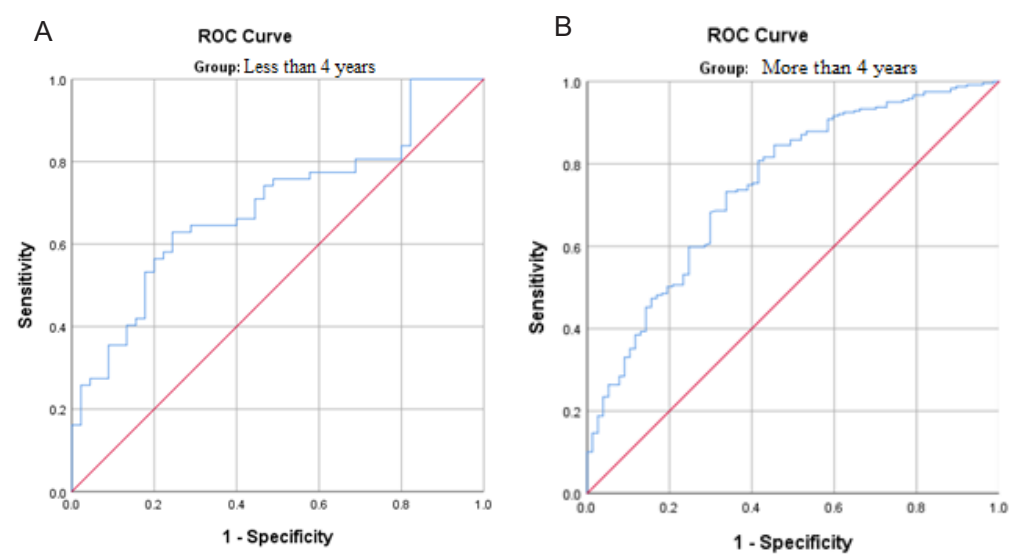

Figure 2. Receiver operating characteristics (ROC) curve diagnostic value of neutrophil to lymphocyte ratio in (A) less than 4 and (B) more than 4 years' pediatrics suspect to acute appendicitis

the cut-off value of 2.3 for patients less than four years. High value of NLR was found statistically associated with acute appendicitis which was diagnosed pathologically. Additionally, the results of this study are consistent with the study of Sevim et al (8).

NLR is a measure of the left-sided shift in white cell production that accompanies the acute inflammatory response. During acute appendicitis it is this increased neutrophil production and its subsequent infiltration of the appendix that drives the acute inflammatory cascade. Previous studies have shown the value of total leukocyte count as an earlier marker of appendiceal inflammation (8, 9). Further studies have demonstrated that a combination of raised total white cell count, with a raised percentage of neutrophils or CRP, has increased sensitivity in diagnosing appendicitis than either variable on its own (9-12).

It is the subtleties in white cell count differentiation that demonstrate acute inflammation and, furthermore, with the appropriate history and examination can aid the diagnosis of acute appendicitis.

Białas and colleagues considered 469 individuals operated for acute appendicitis retrospectively. They found NLR cut off rate as 3.5 similar to our investigation (12).

Cornelis et al demonstrated accessibility of lymphocytopenia as an infectious urgent diagnose in their research, and assumed that NLR was capable to suppose bacteriemia better.

Terradas and colleagues tested 2311 individuals by eosinophil and NLR as a prognostic parameter for bacteriemia in a retrospective cohort investigation. They showed low-eosinophil count, and high values of NLR (>7) as independent risk factors for mortality (13).

Cook et al examined one hundred individuals of suspected to acute appendicitis, and showed a considerable association between NLR ( $\geq 9.3$ postoperative $1^{\text {st }}$ day) (14).

\section{Conclusion}

Acute appendicitis remains a clinical diagnosis, with laboratory and radiological investigations only serving to support the diagnosis. This study has attempted to analyze the available laboratory investigations in order to determine which tests serve to aid this diagnosis. These tests included in the analysis are inexpensive and already part of the routine investigations for patients presenting with abdominal pain. This analysis demonstrates that NLR, in the context of appropriate clinical assessment of patients with a high a priori probability of appendicitis, has a greater diagnostic accuracy in supporting the diagnosis of appendicitis.

\section{Limitations of the study}

One of the limitations of the present study was the small sample size. It is suggested to use long-term studies.

\section{Authors' contribution}

$E P$ and $A N$ and $A B$ were the principal investigators of the study. $E B$ and $A B$ were included in preparing the concept and design. $E P$ and $A N$ revised the manuscript and critically evaluated the intellectual contents. All authors participated in preparing the final draft of the manuscript, revised the manuscript and critically evaluated the intellectual contents. All authors have read and approved the content of the manuscript and confirmed the accuracy or integrity of any part of the work.

\section{Conflicts of interest}

The authors declare that they have no competing interests.

\section{Ethical considerations}

Ethical issues (including plagiarism, data fabrication, double publication) have been completely observed by the authors.

\section{Funding/Support}

This study was supported by Isfahan University of Medical Sciences.

\section{References}

1. Kaiser M, Schroeckenfuchs M, Castellani C, Warncke G, Till $\mathrm{H}$, Singer G. The diagnostic value of hepcidin to predict the presence and severity of appendicitis in children. J Surg Res. 2018;222:102-7. doi: 10.1016/j.jss.2017.10.021.

2. Yousef Y, Youssef F, Dinh T, Pandya K, Stagg H, Homsy M, et al. Risk stratification in pediatric perforated appendicitis: Prospective correlation with outcomes and resource 
utilization. J Pediatr Surg. 2018;53:250-5. doi: 10.1016/j. jpedsurg.2017.11.023.

3. Bonadio W. Time to Appendectomy and Risk of Complicated Appendicitis and Adverse Outcomes in Children. JAMA Pediatr. 2018;172:94-. doi: 10.1001/jamapediatrics.2017.4095.

4. Bekdas M, Ozturk H, Goksugur S, Demircioglu F, Tahaoglu M. Neutrophil to lymphocyte ratio in diagnosis of complicated and non-complicated appendicitis. Sri Lanka J Child Health. 2017;46(1)

5. Salö M, Friman G, Stenström $P$, Ohlsson B, Arnbjörnsson E. Appendicitis in children: evaluation of the pediatric appendicitis score in younger and older children. Surg Res Prec. 2014;2014. doi: 10.1016/j.amjsurg.2019.04.018.

6. Andersson MN, Andersson RE. Causes of short-term mortality after appendectomy: a population-based casecontrolled study. Ann Surg. 2011;254:103-7. doi: 10.1097/ SLA.0b013e31821ad9c4.

7. Chen $\mathrm{C}-\mathrm{Y}$, Chen $\mathrm{Y}-\mathrm{C}, \mathrm{Pu} \mathrm{H}-\mathrm{N}$, Tsai C-H, Chen $\mathrm{W}-\mathrm{T}$, Lin $\mathrm{C}-\mathrm{H}$. Bacteriology of acute appendicitis and its implication for the use of prophylactic antibiotics. Surg Infect. 2012;13:383-90. doi: 10.1089/sur.2011.135.

8. Sevim Y, Namdaroglu O, Akpınar M, Ertem A. Akut Apandisitte Nötrofil Lenfosit Oranının Tanısal Değeri . Sakarya Tıp Dergisi 2014;4:78-81. doi: 10.5505/sakaryamj.2014.29863

9. Shimizu T, Ishizuka M, Kubota K. A lower neutrophil to lymphocyte ratio is closely associated with catarrhal appendicitis versus severe appendicitis. Surgery today. 2016;46:84-10.9. doi: 10.1007/s00595-015-1125-3.

10. Sack U, Biereder B, Elouahidi T, Bauer K, Keller T, Tröbs R-B. Diagnostic value of blood inflammatory markers for detection of acute appendicitis in children. BMC Surg. 2006;6(1):15. doi: 10.1186/1471-2482-6-15.

11. Peltola H, Ahlqvist J, Rapola J, Räsänen J, Louhimo I, Saarinen $\mathrm{M}$, et al. C-reactive protein compared with white blood cell count and erythrocyte sedimentation rate in the diagnosis of acute appendicitis in children. Acta Chirurg Scandinavica. 1986;152:55-8.

12. Białas M, Taran K, Gryszkiewicz M, Modzelewski B. Evaluation of neutrophil-lymphocyte ratio usefulness in the diagnosis of appendicitis. Wiadomosci Lekarskie. 2006;59:601.

13. Terradas R, Grau S, Blanch J, Riu M, Saballs P, Castells X, et al. Eosinophil count and neutrophil-lymphocyte count ratio as prognostic markers in patients with bacteremia: a retrospective cohort study. PloS One. 2012;7:e42860. doi: 10.1371/journal. pone.0042860.

14. Cook EJ, Walsh SR, Farooq N, Alberts JC, Justin TA, Keeling NJ. Post-operative neutrophil-lymphocyte ratio predicts complications following colorectal surgery. Inter J Surg. 2007;5:27-30. doi: 10.1016/j.ijsu.2006.05.013 